\title{
An optimum design of the magnetic circuit of a PM linear electrical generator for the exploitation of sea waves.
}

\author{
Vittorio CECCONI and Marco TRAPANESE, Member, IEEE
}

\begin{abstract}
In this paper an approach to the optimization of the magnetic design of a Permanent Magnet (PM) linear generator for the exploitation of the energy contained in sea waves is presented. This approach consists of a two step procedure: the first one takes into account the stochastic features of the sea wave motion and describe the working condition of the machine under this motion by adopting a lumped parameter model. In this step the objective function is the energy production. The second step optimizes the generator by using the results obtained in the first step as the constraints that must be fulfilled by a design based on a Finite Element Method (FEM) analysis. Furthermore, the approach is applied to design and build a PM linear generator. Some experimental tests carried on the built linear generator are presented.
\end{abstract}

Index Terms-Sea Wave energy converter, PM linear Machines, Synchronous Machine

\section{INTRODUCTION}

A mongst renewable energy sources, sea waves are definitely the one that has been less utilised. This is probably caused by three reasons: i) the low energy concentration in the sea waves, at least in the sites where it is technically possible to install conversion facilities; ii)the strong technical novelties that are required to extract the energy that is contained in a highly and stochastically variable motion; iii) the difficulty to find a suitable electrical system able to convert the energy contained in the motion of the sea into electrical energy. Up to now, the main approach, that has been followed to exploit the energy of sea waves, uses Well's turbines in oscillating water columns (OWC). Recently, the use of linear generator has been proposed to extract energy from sea waves[1-3]. In this approach the slider of a linear generator is directly coupled to a device that is vertically moved from the sea waves. According to the conversion strategy chosen, this device can be a buoyant or an air filled chamber completely submerged. Regardless to which strategy is chosen, wave energy conversion produces high force and low reciprocating speed. Moreover, the behaviour of both of them is highly stochastic because it is caused by the sea waves, which are essentially stochastic.The "main stream" approach to the design of the electromagnetic generator to be used to extract the energy

Manuscript received March 31, 2006. This work was supported in part MIUR and Palermo University.V. Cecconi is with the Electrical Engineering Department (telephone: +390916615265, e-mail: Cecconi@diepa.unipa.it). M.Trapanese is with the Electrical Engineering Department (telephone: +390916615265, e-mail: mtrap@diepa.unipa.it). usually optimises it for one specific working condition[4]. Recently, novel interesting topologies have been proposed [5], but, generally speaking, little attention has been paid to the stochastic features of the phenomena, but in order to maximize the energy extraction, the stochastic features must be included in the mathematical model of the system. In this paper an approach to the optimization of the magnetic design of a PM linear generator is presented. This approach consists of a two step procedure: the first one takes into account the stochastic features of the sea wave motion and describe the working condition of the machine under this motion by adopting a lumped parameter model. In this step the objective function is the energy production. The second step optimizes the generator by using the results obtained in the first step as the constraints that must be fulfilled by a design based on a Finite Element Method (FEM) analysis.

The paper is organized by presenting the optimization procedure in chapter 2 , the machine obtained by using the results of the optimization procedure on chapter 3 and finally the results of some tests on this machine in chapter 4 . In chapter 5 the conclusions are drawn.

\section{OPTIMIZATION PROCEDURE}

As already said, in the following the two steps of the procedure are explained.

\section{II.1 Optimization procedure - First step}

It is assumed that the conversion system has the structure of fig.1. Other conversion topologies can be realized but the one of fig. 1 captures the essential features of the most of conversion systems.

In this device the slider of the PM linear generator is driven from a buoyant (that is rigidly connected to the slider) and counteracted by a spring.

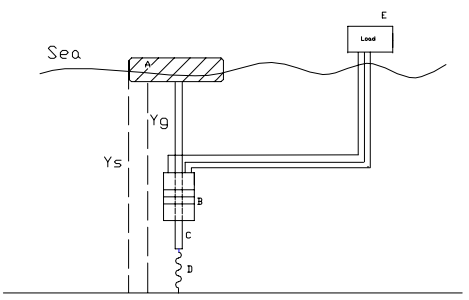

Fig.1 Schematic description of a conversion system based on a PM linear generator. $\mathrm{A}$ is the buoyant, $\mathrm{B}$ is the $\mathrm{PM}$ linear generator, $\mathrm{C}$ is the slider, D is the spring and $\mathrm{E}$ is the electrical load.

The motion of the slider can be modelled as follows: 


$$
\begin{gathered}
\rho g S\left(y_{s-} y_{g}\right)-m g-k i B \\
-k_{s}\left(y_{g}-y_{0}\right)=m \frac{d^{2} y_{g}}{d t^{2}} \\
e(t)=i R_{i}+L \frac{\partial i}{\partial t}+i R \\
e(t)=-\frac{d \varphi}{d t}
\end{gathered}
$$

Where $\mathrm{g}$ is the acceleration gravity, $\rho$ is the water density, $\mathrm{S}$ is the area of the buoyant, $\mathrm{y}_{\mathrm{s}}$ is the vertical coordinate describing the sea level, $y_{g}$ is the vertical coordinate describing the position of the slider, $\mathrm{k}_{\mathrm{s}}$ is the spring constant, $\mathrm{m}$ is total mass of the translator and of the buoyant, $\mathrm{k}$ is the electromagnetic constant, $\mathrm{i}$ the current $\mathrm{B}$ the magnetic induction, $\mathrm{e}(\mathrm{t})$ is the induced electromotive force (emf), $\mathrm{R}_{\mathrm{i}}$ is the internal resistance of the machine, $\mathrm{L}$ is the inductance of the machine, $\mathrm{R}$ is the resistance of the external load, $\varphi$ is the linked flux and $\mathrm{y}_{0}$ is the rest position. The definition of the coordinates can be seen in fig. 1 .

The first equation models the dynamic behaviour of the device. On the left hand side there are the forces acting on the slider and on the right hand side there is the acceleration of the slider. No hydraulic friction is considered

The second equation describes in terms of lumped parameters the generator connected to a resistive load.

The third equations describes the emf.

It is assumed that sea waves can be modelled as follows:

$$
y_{s}=A \sin (\omega t)+y_{0}
$$

where $\mathrm{A}$ is the amplitude and $\omega$ is the frequency. In order to model the stochastic features of the sea wave motion, A and $\omega$ are considered stochastic variables characterized each by a Gaussian distribution.

Several assumptions have been done:

the mathematical model used is one-dimensional;

no losses are assumed in the hydraulic conversion systems;

the losses in the electromagnetic generator are described by a single resistance;

the external load is considered purely resistive (in fact the external load will be buffered by a power electronics device).

In order to optimize the design, several simulation were done. In each simulation parameters $\mathrm{R}_{\mathrm{i}}, \mathrm{L}, \mathrm{k}$ and $\varphi$ had values different from the ones used in the previous simulation. In each run of the simulation the water was let vary according to (2) and a wave train was generated. The wave train consisted of ten oscillations. Each oscillation of the train wave had an amplitude and a frequency generated through a Gaussian generator. $\mathrm{R}_{\mathrm{i}}, \mathrm{L}, \mathrm{k}$ and $\varphi$ were let vary among some values that were compatible with the size of the linear generator, with the induced emf and with the linked flux.

The objective function was the energy output, that under the assumptions presented has the following form:

$$
E_{o}=\int_{0}^{T_{f i n}} i^{2} \cdot R d t
$$

where $E_{o}$ is the output energy and $T_{\text {fin }}$ is the final time. The final $\mathrm{T}$ could vary from run to run and it was equal to the time needed to let the water complete 10 full oscillations.

The output of the first step of the optimisation procedure was the set of the above mentioned parameters that led to the maximum of (3).

\section{II.1 Optimization procedure - second step}

The second step of the optimisation procedure consists of designing a PM linear generator whose parameters were equal or almost equal (within a $5 \%$ ) to the ones obtained in the first step.

A FEM solver, that allows to parametrically design the machine was used. The design proceeded by starting from a first trial design that roughly provided the required parameters of the machine and letting vary in the following trial some geometric parameters randomly within some limits. The adjustable parameters were the pole pitch, the number of coils per pole per phase, the air gap and the number of phases. The length of the slider and the number of poles were fixed. The obtained design was accepted if $R_{i}, L$, $\mathrm{k}$ and $\varphi$ were in agreement with the values obtained in the first step. Among all the designs generated was accepted the design that minimized losses as calculated by the FEM solver.

The losses taken into account were the ohmic losses in the copper and the losses in the iron caused from eddy currents.

Two designs were obtained: one was a single phase machine the other one was a three phase machine. The two designs differed very slightly as far as the magnetic circuit was concerned, as a result only one type of magnetic circuit was chosen. The obtained output parameters are reported in table 1 .

TABLE 1

CHARACTERISTICS OF THE PM GENERATOR OBTAINED

\begin{tabular}{ll}
\hline \hline & \\
Geometric Parameter & Value \\
\hline Pole pitch (mm) & 150 \\
Slot height & 70 \\
Number of phases & $1 / 3$ \\
Number of slots per pole per phase & $3 / 1$ \\
Stator slot width (mm) & 12.5 \\
Tooth height (mm) & 12.5 \\
Stator yoke height (mm) & 60 \\
Length of the magnet $(\mathrm{mm})$ & 50 \\
\hline
\end{tabular}

Along with the design, the behaviour of the no load and loaded emf was obtained. The obtained machine has the geometric parameters that are proportional to the ones of 
the machine presented in [5].

\section{THE OBTAINED MACHINE.}

As already said, two designs were obtained: one was a single phase double sided machine the other one was a three phase double sided machine. The two designs differed very slightly as far as the magnetic circuit was concerned. As a result, a reconfigurable machine was built. In this machine the pole pitch and the slot width were fixed but the number of phases were changeable from 1 to 3 . Also the overall length and of the stator was changeable. In the machine assembled the stator surface was $0.042 \mathrm{~m}^{2}$.

In fig. 2 a picture of a half of stator and a cross section of the laminated modules used in the stator are shown.
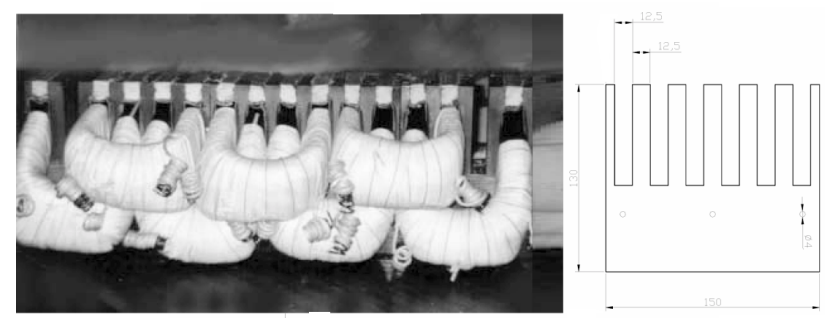

Fig. 2 A) Half of the stator of the machine and B) cross section of one of modules that compose the stator.

\section{THE EXPERIMENTAL RESULTS}

Several tests were performed. All tests were performed in a test bench in laboratory but the machine was not and has not yet been tested in water.

The tests measured the no-load and the loaded emf under various speed slider. In fig. 3 the no load emf in the single phase PM generator is shown for an average speed of 1.6 $\mathrm{m} / \mathrm{s}$.

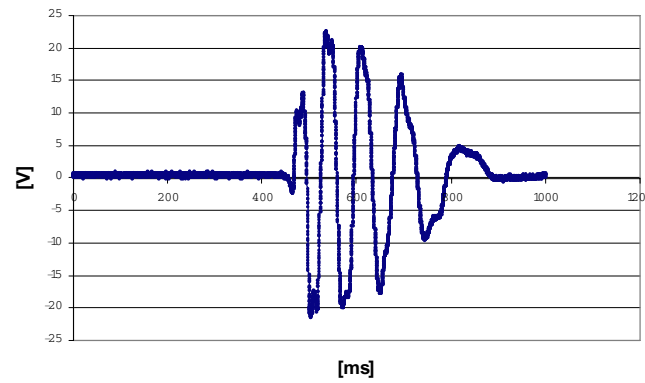

Fig. 3 No load emf in the single phase PM generator. The slider speed is $1.6 \mathrm{~m} / \mathrm{s}$.

This speed was kept during a single run of the slider on the armature. It corresponds to the simulation of half period.

In fig. 4 the no load emf in the three- phase PM generator is shown for a speed of $1.8 \mathrm{~m} / \mathrm{s}$.

It is worth saying how the emf in the single phase case reaches a higher value.In fig. 5 the emf in the single phase PM generator loaded by a resistance $(56 \Omega)$ is shown for a speed of $3.75 \mathrm{~m} / \mathrm{s}$. In fig. 6 the emf in the three- phase PM generator loaded by a three phase resistance $(18 \Omega)$ is shown for a speed of $3.8 \mathrm{~m} / \mathrm{s}$. It is worth saying how in both cases the emf reaches quite a low voltage level. The experimental peak value of the no-load emf and of the loaded emf differed from the designed one for less than $10 \%$.

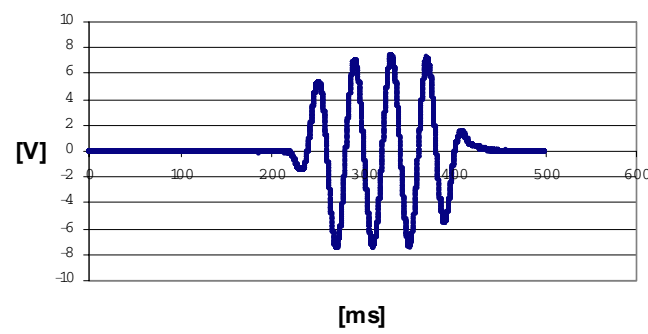

Fig.4 No load in the three phase PM generator. The slider speed is 1.8 $\mathrm{m} / \mathrm{s}$.

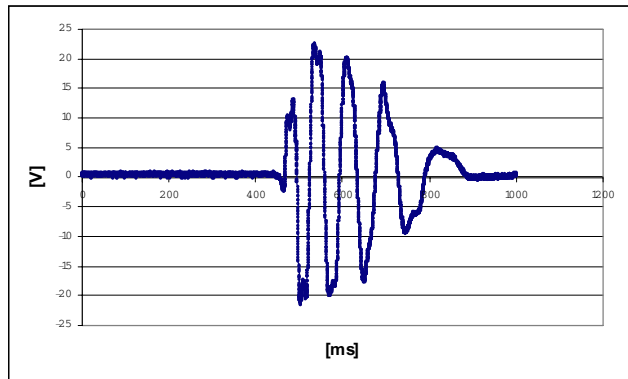

Fig. 5 Loaded emf in the single phase PM generator. The slider speed is $3.75 \mathrm{~m} / \mathrm{s}$.

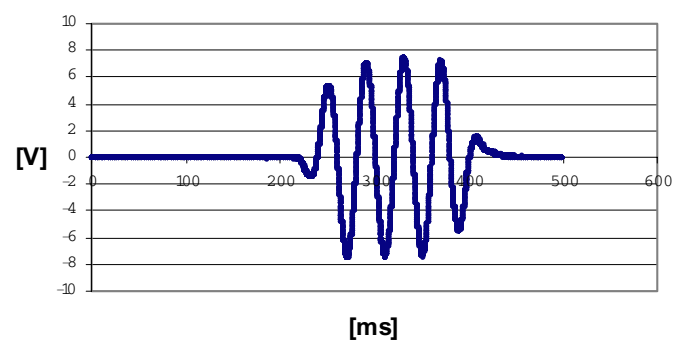

Fig.6 Loaded emf in the three phase PM generator. The slider speed is 3.8 $\mathrm{m} / \mathrm{s}$.

\section{CONCLUSION}

An optimisation design procedure, which includes the stochastic feature of sea wave motion, for a PM linear generator for the explotation of sea waves motion, has been presented. The procedure has been used to design a linear generator. The obtained design has been used to build a generator that was tested in laboratory. The experimental results show that the design approach is reliable with an accuracy of $10 \%$ and that the experimental values are in good agreement with the forecasted ones at least in laboratory tests. Tests on field are needed in order to verify that the machine is able to produce under stochastic conditions.

\section{ACKNOWLEDGMENT}

This work was supported in part by MIUR and Palermo University

\section{REFERENCES}

[1] H. Polinder, F. Gardner, and B. Vriesema "Linear PM generator for wave energy conversion in the AWS" in Proc.14 ${ }^{\text {th }}$ Int. Conf. Electrcial Machines, Helsinki, Finland, 2000, pp. 309-313.

[2] M.A. Mueller, "Electrical generators for direct drive wave energy conversion" in Proc. Inst. Elect. Eng. Gen. Transm. Distr, vol.149, 2002, pp.446-456. 
[3] M. Trapanese, "Un generatore lineare sincrono per lo sfruttamento dell'energia delle onde marine", in Quaderni del Dipartimento di Ingegneria Elettrica dell'Università di Palermo, 1999

[4] H.Polinder, B.C. Mecrow, A.G. Jack, P.G. Dickinson and M.A. Mueller, "Conventionale and TPFM Linear Generators for DirectDrive Wave Energy Conversion", IEEE Transactions on Energy Conversion vol. 20, N.2,Page(s): 219- 224, 2005.

[5] M. Leijon, H. Bernhoff, O. Agren, J. Isberg, J. Sundberg, M. Berg, K.E. Karlsson, A. Wolfbrandt, "Multiphysics simulation of wave energy to electric energy conversion by permanent magnet linear generator", IEEE Transactions on Energy Conversion vol. 20, N.1,Page(s): 260-267, 2005. 